\title{
Looking back and moving forward
}

\author{
The fourth anniversary of Nature Methods' arrival on the publishing scene and a change in \\ leadership offer an opportunity for reflection and editorial fine-tuning.
}

When Nature Methods made its debut in October 2004, just over 4 years ago, it was an anxious but exciting time for our founding chief editor Veronique Kiermer and manuscript editors Nicole Rusk and Daniel Evanko. We were all novices at scientific publishing and more comfortable calibrating a pipette than editing a fledgling journal.

Despite skepticism from some quarters at the time, all of us were convinced that there was an unmet need in the scientific community for a high-profile journal devoted to publishing methodological advances for their own sake.

In the intervening years the scientific community has enthusiastically embraced the journal. Manuscript submissions have steadily increased from 60 a month in the first year to a current monthly average of 125 , and the quality of the typical submission has markedly improved. There is no sign of this trend leveling off either. To help cope with this increase - and the wide range of research submitted to the journal—Allison Doerr and then Natalie de Souza joined the editorial team.

The community interest in the quality methodological papers we have strived to publish has been such that our impact factor has continued to rise, from 6.741 in 2005 to 15.478 in 2007. This could not have occurred without the support of scientists who trusted us with their original research papers and - critically-those who contributed their valuable time and expertise to the peer review process.

Much of the success of Nature Methods has also been due to the tireless efforts of our founding chief editor Veronique Kiermer. But new challenges beckon as Veronique takes on the role of publisher for Nature Methods and Nature Protocols - a position in which she will continue to make a priority of facilitating the dissemination of cutting-edge methodological information-and Daniel Evanko assumes the role of Nature Methods' chief editor.

This change of leadership, combined with the passing of the fourth anniversary of Nature Methods' first issue, provides us with an opportunity to take stock of our content and decide what has gone well and where there is room for improvement.

In our pages we have endeavored to include topics of interest to scientists in all areas of basic biological research, from single-molecule analyses to systems biology; from techniques for virus to human (and everything in between); from advances in established methods like fluorescence imaging and mass spectroscopy to emerging fields like synthetic biology. On one hand, our journal has featured cutting-edge work in areas such as next-generation sequencing and superresolution imaging. On the other hand, we have highlighted advances that do not require sophisticated or expensive equipment but only standard molecular biological tools. We believe this variety is important and will strive to maintain it. Whenever scientific progress calls for it, we will expand into new areas.

In addition to publishing primary research papers, Nature Methods has offered Reviews and Perspectives with a very hands-on focus aimed at providing practical advice for the relative novice in a given field. But the breadth of topics we have covered has not done justice to the diverse interest of our readers, and we are actively working to expand this section.

Another section of the journal to which we intend to devote more resources is the already popular Research Highlights section, where we feature recent methodological advances reported in other journals. To help us gather the most important developments, we welcome your suggestions of recently published papers describing useful methodological developments that you believe merit our readers' attention.

While efforts are being made to expand some sections of the journal, one popular section is being retired. Protocol papers, with detailed step-by-step instructions for established methods, were an important part of Nature Methods when it launched. To expand the scope and volume of published protocols and improve their accessibility, Nature Publishing Group launched Nature Protocols in 2006 as an independent venue for publishing protocols in a convenient online format. Taking advantage of this development, Nature Methods will no longer be publishing Protocols, and the Protocol by Klumperman and colleagues on page 973 will conclude the section. This will allow us to devote more resources to improving other features of Nature Methods.

We hope that our journal has helped dispel the notion that methods are less important than results and deserving only of small print at the end of a paper. Debunking this myth has been and will continue to be our main mission. We will persist in our efforts to bring you, every month, a journal that allows methods to be featured prominently in their own right — as the cornerstones upon which results are based. 